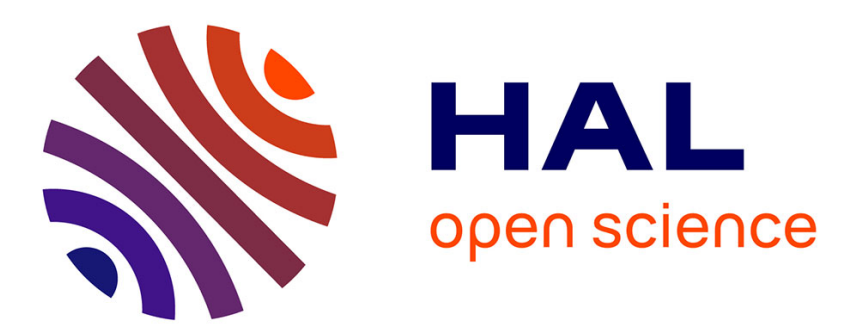

\title{
Optimal unbiased reduced order filtering for discrete-time descriptor systems via LMI
}

Mohamed Darouach, Michel Zasadzinski

\section{To cite this version:}

Mohamed Darouach, Michel Zasadzinski. Optimal unbiased reduced order filtering for discretetime descriptor systems via LMI. Systems and Control Letters, 2009, 58 (6), pp.436-444. 10.1016/j.sysconle.2009.02.005 . hal-00411524

\section{HAL Id: hal-00411524 \\ https://hal.science/hal-00411524}

Submitted on 27 Aug 2009

HAL is a multi-disciplinary open access archive for the deposit and dissemination of scientific research documents, whether they are published or not. The documents may come from teaching and research institutions in France or abroad, or from public or private research centers.
L'archive ouverte pluridisciplinaire HAL, est destinée au dépôt et à la diffusion de documents scientifiques de niveau recherche, publiés ou non, émanant des établissements d'enseignement et de recherche français ou étrangers, des laboratoires publics ou privés. 


\title{
Optimal unbiased reduced order filtering for discrete-time descriptor systems via LMI
}

\author{
M. Darouach and M. Zasadzinski \\ Centre de Recherche en Automatique de Nancy (CRAN), Nancy-Université, CNRS, \\ 186 rue de Lorraine, 54400 Longwy, FRANCE \\ E-mails : darouach@iut-longwy.uhp-nancy.fr, mzasad@iut-longwy.uhp-nancy.fr
}

\begin{abstract}
In this paper, the reduced order unbiased optimal $\mathcal{H}_{\infty}$ filtering problem for discrete-time descriptor linear systems is considered. Necessary and sufficient conditions for the existence of unbiased causal time invariant filters are obtained. Solutions of the filtering problems are given in terms of linear matrix inequalities (LMIs). Parametrization of all optimal causal unbiased filters is provided. The proposed method generalizes the existing results in the standard case.
\end{abstract}

Keywords : Reduced order filter, descriptor systems, unbiased filtering, LMI.

\section{Introduction}

Descriptor systems were introduced to describe the systems for which the standard state-space representation is not applicable. They arise in economics, robotics, electrical and chemical systems $[1,2,3]$. The analysis and the controller design for descriptor systems have received considerable attention in the last decades [3]. As in the standard systems, the knowledge of the state is required for the control or for the failure detection. Full order state estimation for discrete-time descriptor systems has been studied in $[4,5,6,7]$. Recent attention has been concentrated on the $\mathcal{H}_{\infty}$ filtering since, in contrast to the standard Kalman filtering, it does not need any knowledge of the statistical properties of the noise. In the $\mathcal{H}_{\infty}$ filtering problem, the objective is to minimize the energy of the estimation error for the worst-case bounded energy disturbance [8]. It is well known that standard Kalman and $\mathcal{H}_{\infty}$ filters are of order equal to the order of the considered system. In many practical applications, it is often desired to design filters of lower order then those of the systems.

Reduced order Kalman filtering for standard systems was presented in [9] and references therein. On the other hand, [10] developed optimal $\mathcal{H}_{\infty}$ and $\mathcal{L}_{2}-\mathcal{L}_{\infty}$ unbiased reduced order filters for standard systems. Recently [11] has presented a solution to reduced order $\mathcal{H}_{\infty}$ filtering for singular or descriptor systems. They considered the case where the system matrix $E$ is square and may be singular. Their approach does not take into account the unbiasedness of the estimation error.

In this paper, the general case where matrix $E$ may be nonsquare is considered, the order of the filter is equal to the function to be estimated and the unbiasedness of the estimation error is taken into account in the filter design. Our approach is also based on the recently developed bounded real lemma [12]. Its advantage is to use only strict inequalities which leads to tractable and reliable computation. Parametrization of all optimal causal unbiased filters is given. The paper extends the result of [10] to the descriptor system case.

\section{Preliminary results}

In this section we recall some basic results from linear algebra and we present some results on the solvability and estimability which are used in the sequel of the paper. We shall use the following notations. The symbol $\mathcal{R}(A)$ will be used to denote the row space of a matrix $A, \operatorname{Im}(A)=\left\{A x, x \in \mathbb{R}^{n}\right\}$, and 
$\mathcal{R}(A)=\operatorname{Im}\left(A^{T}\right)$. The matrix $A^{\perp}$ denotes a matrix such that $A^{\perp} A^{\perp T}>0$ and $A^{\perp} A=0 . \Sigma^{\dagger}$ denotes any generalized inverse of the matrix $\Sigma$, i.e. verifies $\Sigma \Sigma^{\dagger} \Sigma=\Sigma$.

Let $\Sigma^{\dagger}$ be any generalized inverse of $\Sigma$, then we have the following lemma [13].

Lemma 1. The general solution to $\Sigma X \Sigma=\Sigma$ is given by

$$
X=\Sigma^{\dagger}+V-\Sigma^{\dagger} \Sigma V \Sigma \Sigma^{\dagger}
$$

where $V$ is an arbitrary matrix of appropriate dimension.

Also we have the following lemma proved in standard linear algebra references.

Lemma 2. For any matrices $A \in \mathbb{R}^{m \times n}$ and $L \in \mathbb{R}^{r \times n}, \mathcal{R}(L) \subset \mathcal{R}(A)$ if and only if there exists a matrix $\Phi \in \mathbb{R}^{r \times m}$ such that $L=\Phi A$.

Now consider the following consistent system of linear equations in the variables $x$ and $z$

$$
\begin{aligned}
& A x=b \\
& z=L x
\end{aligned}
$$

where $x \in \mathbb{R}^{n}, z \in \mathbb{R}^{r}$ and $b \in \mathbb{R}^{m}$.

Let the set of solutions of (1a) be defined by $S=\left\{x \in \mathbb{R}^{n}\right.$, such that (1a) is satisfied $\} \neq \emptyset$, then we have the following lemma.

Lemma 3. The following items are equivalent:

1) the vector $z$ is uniquely determined from (1),

2) $\mathcal{R}(L) \subset \mathcal{R}(A)$,

3) $\operatorname{rank}\left[\begin{array}{c}A \\ L\end{array}\right]=\operatorname{rank} A$.

Proof. Let $x_{1}$ be any element of $S$. Assume that $\mathcal{R}(L) \subset \mathcal{R}(A)$, then from lemma 2 there exists a matrix $\Phi$ such that $L=\Phi A$, let $x_{2}$ be another element of $S$, we have $L x_{2}=\Phi A x_{2}=\Phi b=\Phi A x_{1}=L x_{1}=z$, thus $z$ is unique.

Assume that (1) is satisfied, that the value of $z$ is unique for every $x \in S$, then from [13] the value of $x$ is $x=A^{\dagger} b+\left(I_{n}-A^{\dagger} A\right) y$, where $y$ is an arbitrary vector of appropriate dimension. Then $z=L x=$ $L\left(A^{\dagger} b+\left(I_{n}-A^{\dagger} A\right) y\right)$ is unique for every value of $y$ or equivalently $L\left(I_{n}-A^{\dagger} A\right) y=0$ for every $y$ or equivalently $L=L A^{\dagger} A$ which means that $\mathcal{R}(L) \subset \mathcal{R}(A)$.

The proof of the fact that item 2 is equivalent to item 3 is direct.

We have also the following easy to prove lemma.

Lemma 4. Condition 2 of lemma 3 is satisfied if and only if there exists a matrix $J$ such that

$$
J A=\left[\begin{array}{l}
L \\
0
\end{array}\right]
$$

with $\operatorname{dim} J \geqslant(m-\operatorname{rank} A+r) \times m$ and $\operatorname{rank} A \geqslant r$.

The following discrete-time bounded real lemma will be used in this paper.

Lemma 5. [14] Let a stable linear time invariant discrete-time system be described by

$$
\begin{aligned}
& x(t+1)=A x(t)+B w(t) \\
& y(t)=C x(t)+D w(t)
\end{aligned}
$$

with transfer function $G(z)=C(z I-A)^{-1}+D$ and let $\gamma$ be a given positive scalar. Then $\left\|G_{w y}\right\|_{\infty}<\gamma$ if and only if there exists a matrix $P=P^{T}>0$ such that

$$
\left[\begin{array}{ll}
A & B \\
C & D
\end{array}\right]^{T}\left[\begin{array}{ll}
P & 0 \\
0 & I
\end{array}\right]\left[\begin{array}{cc}
A & B \\
C & D
\end{array}\right]<\left[\begin{array}{cc}
P & 0 \\
0 & \gamma^{2} I
\end{array}\right] .
$$


Also we have the following lemma $[14,15]$.

Lemma 6. Let $\mathbb{B}, \mathbb{C}$ and $\mathbb{Q}=\mathbb{Q}^{T}$ be given matrices. Then there exists a matrix $Z$ to solve the matrix inequality

$$
\mathbb{B} Z \mathbb{C}+(\mathbb{B} Z \mathbb{C})^{T}+\mathbb{Q}<0
$$

if and only if the following conditions are satisfied

$$
\mathbb{B}^{\perp} \mathbb{Q B}^{\perp T}<0 \text { and } \quad \mathbb{C}^{T \perp} \mathbb{Q C}^{T \perp T}<0 .
$$

All solutions $\mathcal{Z}$ are given by

$$
\mathcal{Z}=\mathbb{B}_{R}^{\dagger} \mathbb{K} \mathbb{C}_{L}^{\dagger}+\mathbb{Z}-\mathbb{B}_{R}^{\dagger} \mathbb{B}_{R} \mathbb{Z} \mathbb{C}_{L} \mathbb{C}_{L}^{\dagger}
$$

where

$$
\begin{aligned}
\mathbb{K} & =-\mathbb{R}_{1}^{-1} \mathbb{B}_{L}^{T} \mathbb{S}_{1} \mathbb{C}_{R}^{T}\left(\mathbb{C}_{R} \mathbb{S}_{1} \mathbb{C}_{R}^{T}\right)^{-1}+\mathbb{R}_{1}^{-1} \mathbb{S}_{2}^{1 / 2} \mathbb{R}_{2}\left(\mathbb{C}_{R} \mathbb{S}_{1} \mathbb{C}_{R}^{T}\right)^{-1 / 2} \\
\mathbb{S}_{1} & =\left(\mathbb{B}_{L} \mathbb{R}_{1}^{-1} \mathbb{B}_{L}^{T}-\mathbb{Q}\right)^{-1}>0 \\
\mathbb{S}_{2} & =\mathbb{R}_{1}-\mathbb{B}_{L}^{T}\left(\mathbb{S}_{1}-\mathbb{S}_{1} \mathbb{C}_{R}^{T}\left(\mathbb{C}_{R} \mathbb{S}_{1} \mathbb{C}_{R}^{T}\right)^{-1} \mathbb{C}_{R} \mathbb{S}_{1}\right) \mathbb{B}_{L}
\end{aligned}
$$

and $\mathbb{R}_{1}, \mathbb{R}_{2}$ and $\mathbb{Z}$ are arbitrary matrices of appropriate dimensions satisfying $\mathbb{R}_{1}=\mathbb{R}_{1}^{T}>0$ and $\left\|\mathbb{R}_{2}\right\|<1$. Matrices $\mathbb{B}_{L}, \mathbb{B}_{R}, \mathbb{C}_{L}$ and $\mathbb{C}_{R}$ are any full rank matrices such that $\mathbb{B}=\mathbb{B}_{L} \mathbb{B}_{R}$ and $\mathbb{C}=\mathbb{C}_{L} \mathbb{C}_{R}$.

Now let us consider the following discrete-time descriptor system

$$
\begin{aligned}
& E x(t+1)=A x(t)+B w(t) \\
& y(t)=C x(t)+D w(t)
\end{aligned}
$$

where $x(t) \in \mathbb{R}^{n}$ is the semi-state vector, $y(t) \in \mathbb{R}^{p}$ is the measurement output, and $w(t) \in \mathbb{R}^{q}$ is a disturbance vector. $E \in \mathbb{R}^{n \times n}$ and $A \in \mathbb{R}^{n \times n}$ are constant matrices, with rank $E=m_{E} \leqslant n$, and $B \in \mathbb{R}^{n \times q}, C \in \mathbb{R}^{p \times n}$ and $D \in \mathbb{R}^{p \times q}$ are constant matrices.

For $w(t)=0$ we have the following definition [3], [16].

\section{Definition 1.}

1) The pair $(E, A)$ is said to be regular if $\operatorname{det}(z E-A)$ is not identically zero.

2) The pair $(E, A)$ is said to be causal if it is regular and $\operatorname{deg}(\operatorname{det}(z E-A))=\operatorname{rank} E$.

3) The pair $(E, A)$ is said to be stable if it is regular and all roots of $\operatorname{det}(z E-A)=0$ lie inside the unit disc.

4) The pair $(E, A)$ is said to be admissible if it is regular, causal and stable.

We also have the following lemmas.

Lemma 7. [12] The pair $(E, A)$ is admissible if and only if there exist a positive definite matrix $P$ and a symmetric matrix $S$ such that $A^{T}\left(P-E^{\perp T} S E^{\perp}\right) A-E^{T} P E<0$.

Lemma 8. [12] Consider the descriptor system (9). The pair $(E, A)$ is admissible and the transfer matrix $T_{w y}(z)=C(z E-A)^{-1} B$ is $\mathcal{H}_{\infty}$ norm-bounded by a positive real number $\gamma$, i.e. $\left\|T_{w y}\right\|_{\infty}<\gamma$, if and only if there exist a positive definite matrix $P$ and a symmetric matrix $S$ such that

$$
\left[\begin{array}{ll}
\Phi_{11} & \Phi_{12} \\
\Phi_{12}^{T} & \Phi_{22}
\end{array}\right]<0
$$

where

$$
\begin{aligned}
& \Phi_{11}=A^{T}\left(P-E^{\perp T} S E^{\perp}\right) A-E^{T} P E+C^{T} C, \\
& \Phi_{12}=A^{T}\left(P-E^{\perp T} S E^{\perp}\right) B \\
& \Phi_{22}=-\gamma^{2} I_{q}+B^{T}\left(P-E^{\perp T} S E^{\perp}\right) B .
\end{aligned}
$$


In the sequel, without loss of generality, we shall consider the following discrete-time descriptor system

$$
\begin{aligned}
& E x(t+1)=A x(t)+R u(t)+B w(t) \\
& y(t)=C x(t)+D w(t) \\
& z(t)=L x(t)
\end{aligned}
$$

where $x(t) \in \mathbb{R}^{n}$ is the semi-state vector, $y(t) \in \mathbb{R}^{p}$ is the measurement output, $z(t) \in \mathbb{R}^{r}$ is the vector to be estimated, and $w(t) \in \mathbb{R}^{q}$ is a disturbance vector. Matrices $E \in \mathbb{R}^{m \times n}, A \in \mathbb{R}^{m \times n}, B \in \mathbb{R}^{m \times q}$, $C \in \mathbb{R}^{p \times n}, D \in \mathbb{R}^{p \times q}$ and $L \in \mathbb{R}^{r \times n}$ are constant. We assume that $\operatorname{rank} E=m_{E} \leqslant \min (m, n)$, rank $\left[\begin{array}{ll}C & D\end{array}\right]=p$, and that

$$
\operatorname{rank}\left[\begin{array}{cc}
C & D \\
L & 0
\end{array}\right]=\operatorname{rank}\left[\begin{array}{ll}
C & D
\end{array}\right]+\operatorname{rank} L .
$$

Definitions 2 and 3 and theorem 1 on the solvability and estimability are necessary for the sequel of this paper. For these definitions and theorem we assume without loss of generality $w(t)=0$.

Definition 2. The descriptor system (12a) is said to be solvable if there exists $x(t), t \geqslant 0$, solution to (12a).

Definition 3. Let $w(t)=0$. The functional $z(t)$ is said to be estimable for the measurement (12b), if $z(t)$ is uniquely determined by the output $y(t), t \geqslant 0$.

For $w(t)=0$ and $t \in[0, N]$, we can write system (12) in the block matrix form as

$$
\begin{aligned}
& \Phi_{N} X_{N}=Y_{N} \\
& Z_{N}=L_{N} X_{N}
\end{aligned}
$$

where

$$
\begin{aligned}
\Phi_{N}= & {\left[\begin{array}{ccccc}
-A & E & 0 & \cdots & \vdots \\
0 & -A & E & \ddots & \vdots \\
\vdots & \ddots & \ddots & \ddots & 0 \\
0 & \cdots & 0 & -A & E \\
0 & C & 0 & \cdots & 0 \\
0 & 0 & C & \ddots & \vdots \\
\vdots & \ddots & \ddots & \ddots & 0 \\
0 & 0 & \cdots & 0 & C
\end{array}\right], L_{N}=\left[\begin{array}{cccc}
L & 0 & \cdots & 0 \\
0 & L & \ddots & \vdots \\
\vdots & \ddots & \ddots & 0 \\
0 & \cdots & 0 & L
\end{array}\right], Y_{N}=\left[\begin{array}{c}
0 \\
\vdots \\
0 \\
y(1) \\
\vdots \\
y(N-1) \\
y(N)
\end{array}\right], } \\
X_{N}= & {\left[\begin{array}{c}
x(0) \\
x(1) \\
\vdots \\
x(N-1) \\
x(N)
\end{array}\right], \text { and } Z_{N}=\left[\begin{array}{c}
z(0) \\
z(1) \\
\vdots \\
z(N-1) \\
z(N)
\end{array}\right] . }
\end{aligned}
$$

From lemma 3 it is easy to prove the following theorem.

Theorem 1. The functional $z(t)$ is estimable if one of the following assertions are satisfied

1) $\mathcal{R}\left(L_{N}\right) \subset \mathcal{R}\left(\Phi_{N}\right)$,

2) $\mathcal{R}(L) \subset \mathcal{R}\left(\left[\begin{array}{l}E \\ C\end{array}\right]\right)$,

3) $\operatorname{rank}\left[\begin{array}{l}E \\ C \\ L\end{array}\right]=\operatorname{rank}\left[\begin{array}{l}E \\ C\end{array}\right]$. 
Remark 1. If we consider system (12) with $\operatorname{rank} E=m$, then from theorem 1 one can obtain

$$
\operatorname{rank}\left[\begin{array}{cc}
E & A \\
0 & E \\
0 & C \\
0 & L
\end{array}\right]=\operatorname{rank}\left[\begin{array}{cc}
E & A \\
0 & E \\
0 & C
\end{array}\right],
$$

and it is easy to see that when $L=I_{n}$, this condition becomes

$$
\operatorname{rank}\left[\begin{array}{cc}
E & A \\
0 & E \\
0 & C
\end{array}\right]=n+\operatorname{rank} E
$$

which is the impulse observability condition [3].

One can see that condition 3 of theorem 1 generalizes the assumption rank $\left[\begin{array}{l}E \\ C\end{array}\right]=n$ made for the full order filter [4], [5], and [6]. In the sequel of the paper we make the following assumption.

Assumption 1. $\operatorname{rank}\left[\begin{array}{c}E \\ C \\ L\end{array}\right]=\operatorname{rank}\left[\begin{array}{c}E \\ C\end{array}\right]$.

\section{Optimal $\mathcal{H}_{\infty}$ unbiased reduced order filter}

In the present section, a design of an optimal unbiased reduced order filter for system (12) will be developed. Two cases will be considered, the general case and a particular case.

\subsection{General case}

From lemma 4 and under assumption 1 there exists a full row rank matrix $J=\left[\begin{array}{ll}J_{1} & J_{2} \\ J_{3} & J_{4}\end{array}\right]$ such that

$$
\left[\begin{array}{ll}
J_{1} & J_{2} \\
J_{3} & J_{4}
\end{array}\right]\left[\begin{array}{l}
E \\
C
\end{array}\right]=\left[\begin{array}{l}
L \\
0
\end{array}\right]
$$

where $J \in \mathbb{R}^{\ell \times(m+p)}$ with $\ell \geqslant m+p+r-\operatorname{rank}\left[\begin{array}{l}E \\ C\end{array}\right]$.

Consider the following functional $r^{\text {th }}$ order filter for system (12)

$$
\begin{aligned}
& \zeta(t+1)=N \zeta(t)+\bar{\Gamma} u(t)+\Gamma y(t) \\
& \widehat{z}(t)=\zeta(t)+\left(J_{2}-\mathbf{K}_{1} J_{4}\right) y(t)
\end{aligned}
$$

where $\widehat{z}(t) \in \mathbb{R}^{r}$ is the estimate of $z(t)$, matrices $N, \Gamma$, and $\mathbf{K}_{1}$ are of appropriate dimensions and must be determined such that the estimation error $e(t)=z(t)-\widehat{z}(t)$ is unbiased.

Now the estimation error is

$$
e(t)=z(t)-\widehat{z}(t)=L x(t)-\zeta(t)-\left(J_{2}-\mathbf{K}_{1} J_{4}\right) y(t) .
$$

From (12), and under assumption 1 and the definition of matrix $J$, we obtain

$$
e(t)=\left(J_{1}-\mathbf{K}_{1} J_{3}\right) E x(t)-\zeta(t)-\left(J_{2}-\mathbf{K}_{1} J_{4}\right) D w(t)
$$

and its dynamics is given by

$$
\begin{array}{r}
e(t+1)=N e(t)+\left(\left(J_{1}-\mathbf{K}_{1} J_{3}\right) A-N L+\left(N\left(J_{2}-\mathbf{K}_{1} J_{4}\right)-\Gamma\right) C\right) x(t)+\left(\left(J_{1}-\mathbf{K}_{1} J_{3}\right) R-\bar{\Gamma}\right) u(t) \\
+\left(\left(J_{1}-\mathbf{K}_{1} J_{3}\right) B+\left(N\left(J_{2}-\mathbf{K}_{1} J_{4}\right)-\Gamma\right) D\right) w(t)-\left(J_{2}-\mathbf{K}_{1} J_{4}\right) D w(t+1) .
\end{array}
$$


From (16), $\widehat{z}(t)$ is an unbiased estimate of $z(t)$ if and only if

$$
\left[\begin{array}{lll}
N & \mathbf{K}_{1} & \mathbf{K}_{2}
\end{array}\right] \Sigma=\Theta
$$

where $\mathbf{K}_{2}=\Gamma-N\left(J_{2}-\mathbf{K}_{1} J_{4}\right), \Sigma=\left[\begin{array}{c}L \\ J_{3} A \\ C\end{array}\right], \Theta=J_{1} A$ and $\bar{\Gamma}=\left(J_{1}-\mathbf{K}_{1} J_{3}\right) R$.

In this case equation (16) becomes

$$
e(t+1)=N e(t)+\Gamma_{1} w(t)+\Gamma_{2} w(t+1)
$$

where $\Gamma_{1}=J_{1} B-\mathbf{K}_{1} J_{3} B-\mathbf{K}_{2} D$ and $\Gamma_{2}=-J_{2} D+\mathbf{K}_{1} J_{4} D$.

Now equation (17) has a solution if and only if

$$
\operatorname{rank}\left[\begin{array}{c}
\Sigma \\
\Theta
\end{array}\right]=\operatorname{rank} \Sigma
$$

or equivalently

$$
\operatorname{rank}\left[\begin{array}{c}
L \\
J_{1} A \\
J_{3} A \\
C
\end{array}\right]=\operatorname{rank}\left[\begin{array}{c}
L \\
J_{3} A \\
C
\end{array}\right]
$$

Under condition (19), the general solution to (18) is given by

$$
\left[\begin{array}{lll}
N & \mathbf{K}_{1} & \mathbf{K}_{2}
\end{array}\right]=\Theta \Sigma^{\dagger}-\mathcal{Z}\left(I_{\ell+p}-\Sigma \Sigma^{\dagger}\right)
$$

where $\mathcal{Z}$ is an arbitrary matrix of appropriate dimension.

Define the following matrices,

$$
\begin{aligned}
& \mathcal{F}=\Theta \Sigma^{\dagger}\left[\begin{array}{c}
I_{r} \\
0 \\
0
\end{array}\right], \quad \mathcal{G}=\left(I_{\ell+p}-\Sigma \Sigma^{\dagger}\right)\left[\begin{array}{c}
I_{r} \\
0 \\
0
\end{array}\right], \quad \mathcal{F}_{1}=J_{1} B-\Theta \Sigma^{\dagger}\left[\begin{array}{c}
0 \\
J_{3} B \\
D
\end{array}\right], \\
& \mathcal{G}_{1}=\left(I_{\ell+p}-\Sigma \Sigma^{\dagger}\right)\left[\begin{array}{c}
0 \\
J_{3} B \\
D
\end{array}\right], \quad \mathcal{F}_{2}=-J_{2} D+\Theta \Sigma^{\dagger}\left[\begin{array}{c}
0 \\
J_{4} D \\
0
\end{array}\right], \quad \mathcal{G}_{2}=\left(I_{\ell+p}-\Sigma \Sigma^{\dagger}\right)\left[\begin{array}{c}
0 \\
J_{4} D \\
0
\end{array}\right]
\end{aligned}
$$

then matrices $N, \Gamma_{1}$ and $\Gamma_{2}$ can be written as

$$
N=\mathcal{F}-\mathcal{Z} \mathcal{G}, \Gamma_{1}=\mathcal{F}_{1}-\mathcal{Z} \mathcal{G}_{1} \text { and } \Gamma_{2}=\mathcal{F}_{2}-\mathcal{Z} \mathcal{G}_{2} .
$$

On the other hand equation (16) can be rewritten as follows

$$
\begin{aligned}
& \mathcal{E} \bar{x}(t+1)=\mathcal{A} \bar{x}(t)+\mathcal{B} w(t) \\
& e(t)=\mathcal{C} \bar{x}(t)
\end{aligned}
$$

where

$$
\bar{x}(t)=\left[\begin{array}{c}
e(t) \\
w(t)
\end{array}\right], \mathcal{E}=\left[\begin{array}{cc}
I_{r} & -\Gamma_{2} \\
0 & 0
\end{array}\right], \mathcal{A}=\left[\begin{array}{cc}
N & \Gamma_{1} \\
0 & -I_{q}
\end{array}\right], \mathcal{B}=\left[\begin{array}{c}
0 \\
I_{q}
\end{array}\right] \text { and } \mathcal{C}=\left[\begin{array}{ll}
I_{r} & 0
\end{array}\right] .
$$

Now define the following nonsingular matrices

$$
\Omega=\left[\begin{array}{cc}
I_{r} & N \Gamma_{2}+\Gamma_{1} \\
0 & -I_{q}
\end{array}\right] \text { and } \Phi=\left[\begin{array}{cc}
I_{r} & \Gamma_{2} \\
0 & I_{q}
\end{array}\right]
$$

then we obtain

$$
\mathcal{E}_{1}=\Omega \mathcal{E} \Phi=\left[\begin{array}{cc}
I_{r} & 0 \\
0 & 0
\end{array}\right], \mathcal{A}_{1}=\Omega \mathcal{A} \Phi=\left[\begin{array}{cc}
N & 0 \\
0 & I_{q}
\end{array}\right], \mathcal{B}_{1}=\Omega \mathcal{B}=\left[\begin{array}{c}
N \Gamma_{2}+\Gamma_{1} \\
-I_{q}
\end{array}\right] \text { and } \mathcal{C}_{1}=\mathcal{C} \Phi=\left[\begin{array}{ll}
I_{r} & \Gamma_{2}
\end{array}\right] .
$$


It is easy to see that system $(21)$ described by $(\mathcal{E}, \mathcal{A}, \mathcal{B}, \mathcal{C})$ is restrictively equivalent to the one described by $\left(\mathcal{E}_{1}, \mathcal{A}_{1}, \mathcal{B}_{1}, \mathcal{C}_{1}\right)[3]$.

On the other hand, matrix $\mathcal{B}_{1}$ contains the term $N \Gamma_{2}$ which is nonlinear in the parameter matrix $\mathcal{Z}$. To alleviate this nonlinearity we introduce the following matrix parameter $\mathcal{Z}=\overline{\mathcal{Z}}\left(I_{\ell+p}-\mathcal{G}_{2} \mathcal{G}_{2}^{\dagger}\right)$. In this case matrices $N, \Gamma_{1}$ and $\Gamma_{2}$ become

$$
N=\mathcal{F}-\overline{\mathcal{Z}} \overline{\mathcal{G}}, \Gamma_{1}=\mathcal{F}_{1}-\overline{\mathcal{Z}} \overline{\mathcal{G}}_{1} \text { and } \Gamma_{2}=\mathcal{F}_{2}
$$

where $\overline{\mathcal{G}}=\left(I_{\ell+p}-\mathcal{G}_{2} \mathcal{G}_{2}^{\dagger}\right) \mathcal{G}$ and $\overline{\mathcal{G}}_{1}=\left(I_{\ell+p}-\mathcal{G}_{2} \mathcal{G}_{2}^{\dagger}\right) \mathcal{G}_{1}$. Consequently matrices $\mathcal{A}_{1}$, $\mathcal{B}_{1}$ and $\mathcal{C}_{1}$ can be written as

$$
\mathcal{A}_{1}=\mathcal{A}_{e 1}-\left[\begin{array}{c}
I_{r} \\
0
\end{array}\right] \overline{\mathcal{Z}} \mathcal{A}_{e 2}, \mathcal{B}_{1}=\mathcal{B}_{e 1}-\left[\begin{array}{c}
I_{r} \\
0
\end{array}\right] \overline{\mathcal{Z}} \mathcal{B}_{e 2} \text { and } \mathcal{C}_{1}=\left[\begin{array}{ll}
I_{r} & \mathcal{F}_{2}
\end{array}\right]
$$

where $\mathcal{A}_{e 1}=\left[\begin{array}{cc}\mathcal{F} & 0 \\ 0 & I_{q}\end{array}\right], \mathcal{A}_{e 2}=\left[\begin{array}{cc}\overline{\mathcal{G}} & 0\end{array}\right], \mathcal{B}_{e 1}=\left[\begin{array}{c}\mathcal{F} \mathcal{F}_{2}+\mathcal{F}_{1} \\ -I_{q}\end{array}\right]$ and $\mathcal{B}_{e 2}=\overline{\mathcal{G}} \mathcal{F}_{2}+\mathcal{F}_{1}$.

Now the solution of the $\mathcal{H}_{\infty}$ optimal filtering problem for system (12) is then given by the following theorem.

Theorem 2. Under assumption 1 and (19), there exists a parameter matrix $\overline{\mathcal{Z}}$ such that (15) is an unbiased filter for system (12) which solves the $\gamma$-suboptimal $\mathcal{H}_{\infty}$ filtering problem if and only if there exist a positive definite matrix $P_{1}$, a positive definite matrix $Q=\left[\begin{array}{ll}Q_{1} & Q_{2} \\ Q_{2}^{T} & Q_{3}\end{array}\right]$ and a symmetric matrix $S$ such that

$$
\begin{gathered}
{\left[\begin{array}{cc}
P_{1} & I_{r} \\
I_{r} & Q_{1}
\end{array}\right]>0} \\
{\left[\begin{array}{cccc}
I_{r}-P_{1} & \mathcal{F}_{2} & 0 & 0 \\
\mathcal{F}_{2}^{T} & \mathcal{F}_{2}^{T} \mathcal{F}_{2}-S & S & I_{q} \\
0 & S & -S-\gamma^{2} I_{q} & -I_{q} \\
0 & I_{q} & -I_{q} & -Q_{3}
\end{array}\right]<0}
\end{gathered}
$$

and

$$
\left[\begin{array}{cccc}
\mathbb{K}_{1}\left(I_{r}-P_{1}\right) \mathbb{K}_{1}^{T}-\mathbb{K}_{2}\left(S+\gamma^{2} I_{q}\right) \mathbb{K}_{2}^{T} & \mathbb{K}_{1} \mathcal{F}_{2}+\mathbb{K}_{2} S & \mathbb{K}_{1} \mathcal{F}^{T}+\mathbb{K}_{2}\left(\mathcal{F}_{2}^{T} \mathcal{F}^{T}+\mathcal{F}_{1}^{T}\right) & -\mathbb{K}_{2} \\
\mathcal{F}_{2}^{T} \mathbb{K}_{1}^{T}+S \mathbb{K}_{2}^{T} & \mathcal{F}_{2}^{T} \mathcal{F}_{2}-S & 0 & I_{q} \\
\mathcal{F} \mathbb{K}_{1}^{T}+\left(\mathcal{F} \mathcal{F}_{2}+\mathcal{F}_{1}\right) \mathbb{K}_{2}^{T} & 0 & -Q_{1} & -Q_{2} \\
-\mathbb{K}_{2}^{T} & I_{q} & -Q_{2}^{T} & -Q_{3}
\end{array}\right]<0
$$

with $\left[\begin{array}{ll}\mathbb{K}_{1} & \mathbb{K}_{2}\end{array}\right]=\left[\begin{array}{c}\overline{\mathcal{G}}^{T} \\ \mathcal{F}_{2}^{T} \overline{\mathcal{G}}^{T}+\overline{\mathcal{G}}_{1}^{T}\end{array}\right]^{\perp}$.

In this case all parameter matrices $\overline{\mathcal{Z}}$ are given by

$$
\overline{\mathcal{Z}}=\mathbb{B}_{R}^{\dagger} \mathbb{K}_{L}^{\dagger}+\mathbb{Z}-\mathbb{B}_{R}^{\dagger} \mathbb{B}_{R} \mathbb{Z} \mathbb{C}_{L} \mathbb{C}_{L}^{\dagger}
$$

where

$$
\begin{aligned}
\mathbb{K} & =-\mathbb{R}_{1}^{-1} \mathbb{B}_{L}^{T} \mathbb{S}_{1} \mathbb{C}_{R}^{T}\left(\mathbb{C}_{R} \mathbb{S}_{1} \mathbb{C}_{R}^{T}\right)^{-1}+\mathbb{R}_{1}^{-1} \mathbb{S}_{2}^{1 / 2} \mathbb{R}_{2}\left(\mathbb{C}_{R} \mathbb{S}_{1} \mathbb{C}_{R}^{T}\right)^{-1 / 2} \\
\mathbb{S}_{1}= & \left(\mathbb{B}_{L} \mathbb{R}_{1}^{-1} \mathbb{B}_{L}^{T}-\overline{\mathbb{Q}}\right)^{-1}>0 \\
\mathbb{S}_{2}= & \mathbb{R}_{1}-\mathbb{B}_{L}^{T}\left(\mathbb{S}_{1}-\mathbb{S}_{1} \mathbb{C}_{R}^{T}\left(\mathbb{C}_{R} \mathbb{S}_{1} \mathbb{C}_{R}^{T}\right)^{-1} \mathbb{C}_{R} \mathbb{S}_{1}\right) \mathbb{B}_{L} \\
{\left[\begin{array}{ll}
\overline{\mathbb{B}} & \overline{\mathbb{Q}} \\
\bullet & \overline{\mathbb{C}}
\end{array}\right]=} & {\left[\begin{array}{c|ccccc}
0 & I_{r}-P_{1} & \mathcal{F}_{2} & 0 & \mathcal{F}^{T} & 0 \\
0 & \mathcal{F}_{2}^{T} & \mathcal{F}_{2}^{T} \mathcal{F}_{2}-S & S & 0 & I_{q} \\
0 & 0 & S & -S-\gamma^{2} I_{q} & \mathcal{F}_{2}^{T} \mathcal{F}^{T}+\mathcal{F}_{1}^{T} & -I_{q} \\
I_{r} & \mathcal{F} & 0 & \mathcal{F} \mathcal{F}_{2}+\mathcal{F}_{1} & -Q_{1} & -Q_{2} \\
0 & 0 & I_{q} & -I_{q} & -Q_{2}^{T} & -Q_{3} \\
\hline \bullet & \overline{\mathcal{G}} & 0 & \overline{\mathcal{G}} \mathcal{F}_{2}+\overline{\mathcal{G}}_{1} & 0 & 0
\end{array}\right] }
\end{aligned}
$$


and $\mathbb{R}_{1}, \mathbb{R}_{2}$ and $\mathbb{Z}$ are arbitrary matrices of appropriate dimensions satisfying $\mathbb{R}_{1}=\mathbb{R}_{1}^{T}>0$ and $\left\|\mathbb{R}_{2}\right\|<1$. Matrices $\mathbb{B}_{L}, \mathbb{B}_{R}, \mathbb{C}_{L}$ and $\mathbb{C}_{R}$ are any full rank matrices such that $\overline{\mathbb{B}}=\mathbb{B}_{L} \mathbb{B}_{R}$ and $\overline{\mathbb{C}}=\mathbb{C}_{L} \mathbb{C}_{R}$.

Proof. From system (21) and from lemma 8, $\left\|T_{w e}\right\|_{\infty}<\gamma$ if and only if there exist a positive definite matrix $P=\left[\begin{array}{cc}P_{1} & P_{2} \\ P_{2}^{T} & P_{3}\end{array}\right]$ and a symmetric matrix $S$ such that

$$
\left[\begin{array}{ll}
\Phi_{11} & \Phi_{12} \\
\Phi_{12}^{T} & \Phi_{22}
\end{array}\right]<0
$$

where

$$
\begin{aligned}
\Phi_{11} & =\mathcal{A}_{1}^{T}\left(P-\mathcal{E}_{1}^{\perp T} S \mathcal{E}_{1}^{\perp}\right) \mathcal{A}_{1}-\mathcal{E}_{1}^{T} P \mathcal{E}_{1}+\mathcal{C}_{1}^{T} \mathcal{C}_{1}, \\
\Phi_{12} & =\mathcal{A}_{1}^{T}\left(P-\mathcal{E}_{1}^{\perp T} S \mathcal{E}_{1}^{\perp}\right) \mathcal{B}_{1}, \\
\Phi_{22} & =-\gamma^{2} I_{q}+\mathcal{B}_{1}^{T}\left(P-\mathcal{E}_{1}^{\perp T} S \mathcal{E}_{1}^{\perp}\right) \mathcal{B}_{1} .
\end{aligned}
$$

Now, from the expressions of $\mathcal{E}_{1}, \mathcal{A}_{1}$ and $\mathcal{B}_{1}$, we have $\mathcal{E}_{1}^{\perp}=\left[\begin{array}{ll}0 & I_{q}\end{array}\right]=\mathcal{E}_{1}^{\perp} \mathcal{A}_{1}$ and $\mathcal{E}_{1}^{\perp} \mathcal{B}_{1}=-I_{q}$, then matrices $\Phi_{11}, \Phi_{12}$ and $\Phi_{22}$ become

$$
\begin{aligned}
\Phi_{11} & =\mathcal{A}_{1}^{T} P \mathcal{A}_{1}-\mathcal{E}_{1}^{\perp T} S \mathcal{E}_{1}^{\perp}-\mathcal{E}_{1}^{T} P \mathcal{E}_{1}+\mathcal{C}_{1}^{T} \mathcal{C}_{1} \\
\Phi_{12} & =\mathcal{A}_{1}^{T} P \mathcal{B}_{1}+\mathcal{E}_{1}^{\perp T} S, \\
\Phi_{22} & =-\gamma^{2} I_{q}+\mathcal{B}_{1}^{T} P \mathcal{B}_{1}-S,
\end{aligned}
$$

and inequality (27) can be written as

$$
\left[\begin{array}{cc}
\mathcal{A}_{1}^{T} P \mathcal{A}_{1}-\mathcal{E}_{1}^{\perp T} S \mathcal{E}_{1}^{\perp}-\mathcal{E}_{1}^{T} P \mathcal{E}_{1}+\mathcal{C}_{1}^{T} \mathcal{C}_{1} & \mathcal{A}_{1}^{T} P \mathcal{B}_{1}+\mathcal{E}_{1}^{\perp T} S \\
\left(\mathcal{A}_{1}^{T} P \mathcal{B}_{1}+\mathcal{E}_{1}^{\perp T} S\right)^{T} & -\gamma^{2} I_{q}+\mathcal{B}_{1}^{T} P \mathcal{B}_{1}-S
\end{array}\right]<0
$$

or equivalently, by using the Schur lemma,

$$
\left[\begin{array}{ccc}
-\mathcal{E}_{1}^{\perp T} S \mathcal{E}_{1}^{\perp}-\mathcal{E}_{1}^{T} P \mathcal{E}_{1}+\mathcal{C}_{1}^{T} \mathcal{C}_{1} & \mathcal{E}_{1}^{\perp T} S & \mathcal{A}_{1}^{T} P \\
S \mathcal{E}_{1}^{\perp} & -S-\gamma^{2} I_{q} & \mathcal{B}_{1}^{T} P \\
P \mathcal{A}_{1} & P \mathcal{B}_{1} & -P
\end{array}\right]<0
$$

From the expressions of $\mathcal{A}_{1}$ and $\mathcal{B}_{1}$, and by pre- and post-multiplying inequality (28) by $\left[\begin{array}{ccc}I_{r+q} & 0 & 0 \\ 0 & I_{q} & 0 \\ 0 & 0 & Q\end{array}\right]$, we obtain the following LMI

$$
\overline{\mathbb{Q}}+\left[\begin{array}{c}
0 \\
0 \\
I_{r} \\
0
\end{array}\right] \overline{\mathcal{Z}}\left[\begin{array}{llll}
\overline{\mathcal{G}} & 0 & \overline{\mathcal{G}} \mathcal{F}_{2}+\overline{\mathcal{G}}_{1} & 0
\end{array}\right]+\left[\begin{array}{llll}
\overline{\mathcal{G}} & 0 & \overline{\mathcal{G}} \mathcal{F}_{2}+\overline{\mathcal{G}}_{1} & 0
\end{array}\right]^{T} \overline{\mathcal{Z}}^{T}\left[\begin{array}{c}
0 \\
0 \\
I_{r} \\
0
\end{array}\right]^{T}<0
$$

where

$$
\overline{\mathbb{Q}}=\left[\begin{array}{ccc}
-\mathcal{E}_{1}^{\perp T} S \mathcal{E}_{1}^{\perp}-\mathcal{E}_{1}^{T} P \mathcal{E}_{1}+\mathcal{C}_{1}^{T} \mathcal{C}_{1} & \mathcal{E}_{1}^{\perp T} S & \mathcal{A}_{e 1}^{T} \\
S \mathcal{E}_{1}^{\perp} & -S-\gamma^{2} I_{q} & \mathcal{B}_{e 1}^{T} \\
\mathcal{A}_{e 1} & \mathcal{B}_{e 1} & -Q
\end{array}\right]
$$

and

$$
Q=\left[\begin{array}{ll}
Q_{1} & Q_{2} \\
Q_{2}^{T} & Q_{3}
\end{array}\right]=\left[\begin{array}{ll}
P_{1} & P_{2} \\
P_{2}^{T} & P_{3}
\end{array}\right]^{-1}
$$

Due to $(30)$ and $P=P^{T}>0$, we have [15]

$$
\left[\begin{array}{cc}
P_{1} & I_{r} \\
I_{r} & Q_{1}
\end{array}\right]>0
$$


Using lemma 6 we obtain

$$
\left[\begin{array}{c}
0 \\
0 \\
I_{r} \\
0
\end{array}\right]^{\perp} \overline{\mathbb{Q}}\left[\begin{array}{c}
0 \\
0 \\
I_{r} \\
0
\end{array}\right]^{\perp T}
$$

and

$$
\left[\begin{array}{llll}
\overline{\mathcal{G}} & 0 & \overline{\mathcal{G}} \mathcal{F}_{2}+\overline{\mathcal{G}}_{1} & 0
\end{array}\right]^{T \perp} \overline{\mathbb{Q}}\left[\begin{array}{llll}
\overline{\mathcal{G}} & 0 & \overline{\mathcal{G}} \mathcal{F}_{2}+\overline{\mathcal{G}}_{1} & 0
\end{array}\right]^{T \perp T}<0 .
$$

Inequalities (23) and (24) are obtained by substituting

$$
\left[\begin{array}{c}
0 \\
0 \\
I_{r} \\
0
\end{array}\right]^{\perp}=\left[\begin{array}{ccc}
I_{r+q} & 0 & 0 \\
0 & I_{q} & 0 \\
0 & 0 & \mathcal{E}_{1}^{\perp}
\end{array}\right] \text { and }\left[\begin{array}{c}
\overline{\mathcal{G}}^{T} \\
0 \\
\mathcal{F}_{2}^{T} \overline{\mathcal{G}}^{T}+\overline{\mathcal{G}}_{1}^{T} \\
0
\end{array}\right]^{\perp}=\left[\begin{array}{cccc}
\mathbb{K}_{1} & 0 & \mathbb{K}_{2} & 0 \\
0 & I_{q} & 0 & 0 \\
0 & 0 & 0 & I_{r+q}
\end{array}\right]
$$

in inequalities (32) and (33) respectively, with $\left[\begin{array}{ll}\mathbb{K}_{1} & \mathbb{K}_{2}\end{array}\right]=\left[\begin{array}{c}\overline{\mathcal{G}}^{T} \\ \mathcal{F}_{2}^{T} \overline{\mathcal{G}}^{T}+\overline{\mathcal{G}}_{1}^{T}\end{array}\right]^{\perp}$.

Equations (25) and (26) are deduced from relations $(7)$ and (8) of lemma 6 by replacing $\mathcal{Z}$ by $\overline{\mathcal{Z}}$.

Theorem 2 reveals that the $\gamma$-suboptimal $\mathcal{H}_{\infty}$ unbiased reduced order filtering for system (12) can be obtained by solving an LMI problem, since inequalities (22), (23) and (24) are linear on the variables $P_{1}>0$ and $Q$.

Now, we can give the following remarks concerning condition (19) and the invariance of the filter to the choice of the generalized inverse.

Remark 2. Condition (19) generalizes the existing results in the standard systems (see condition (11) of reference [10]). In fact when $E=I_{n}$, one choice of the matrix $J$ is $J=\left[\begin{array}{ll}J_{1} & J_{2} \\ J_{3} & J_{4}\end{array}\right]=\left[\begin{array}{cc}L & 0 \\ -C & I_{p}\end{array}\right]$, which leads to $J\left[\begin{array}{c}I_{n} \\ C\end{array}\right]=\left[\begin{array}{c}L \\ 0\end{array}\right]$. In this case condition (19) can be written as rank $\left[\begin{array}{c}L \\ L A \\ C A \\ C\end{array}\right]=\operatorname{rank}\left[\begin{array}{c}L \\ C A \\ C\end{array}\right]$ which corresponds to the condition given by [17] which is more general than that given by [10].

When the matrix $L=I_{n}$, the matrix $J$ is nonsingular and condition (19) becomes $\operatorname{rank}\left[\begin{array}{c}I_{n} \\ J_{1} A \\ J_{3} A \\ C\end{array}\right]=$ $\operatorname{rank}\left[\begin{array}{c}I_{n} \\ J_{3} A \\ C\end{array}\right]=n$ which is always satisfied.

From these remarks it is seen that condition (19) is not restrictive and it generalizes the existing conditions for the standard systems.

Remark 3. The design of the filter (15) is independent of the choice of the generalized inverse $\Sigma^{\dagger}$. This can be proved as follows. Assume that equation (17) has a solution, i.e. that condition (19) holds or, equivalently, that $\Theta \Sigma^{\dagger} \Sigma=\Theta$.

Now let $X$ be the general solution of $\Sigma X \Sigma=\Sigma$, from lemma 1 we have $X=\Sigma^{\dagger}+V-\Sigma^{\dagger} \Sigma V \Sigma \Sigma^{\dagger}$ then by using this value as a generalized inverse in matrix $N$ we obtain 


$$
\begin{aligned}
N & =\widetilde{F}-\mathcal{Z} \widetilde{G}=\Theta X\left[\begin{array}{c}
I_{r} \\
0 \\
0
\end{array}\right]-\mathcal{Z}\left(I_{\ell+p}-\Sigma X\right)\left[\begin{array}{c}
I_{r} \\
0 \\
0
\end{array}\right] \\
& =\Theta\left(\Sigma^{\dagger}+V-\Sigma^{\dagger} \Sigma V \Sigma \Sigma^{\dagger}\right)\left[\begin{array}{c}
I_{r} \\
0 \\
0
\end{array}\right]-\mathcal{Z}\left(I_{\ell+p}-\Sigma\left(\Sigma^{\dagger}+V-\Sigma^{\dagger} \Sigma V \Sigma \Sigma^{\dagger}\right)\right)\left[\begin{array}{c}
I_{r} \\
0 \\
0
\end{array}\right] \\
& =\left(\Theta \Sigma^{\dagger}+\Theta V-\Theta \Sigma^{\dagger} \Sigma V \Sigma \Sigma^{\dagger}\right)\left[\begin{array}{c}
I_{r} \\
0 \\
0
\end{array}\right]-\mathcal{Z}\left(I_{\ell+p}-\Sigma \Sigma^{\dagger}+\Sigma V-\Sigma V \Sigma \Sigma^{\dagger}\right)\left[\begin{array}{c}
I_{r} \\
0 \\
0
\end{array}\right] \\
& =\left(\Theta \Sigma^{\dagger}+\Theta V-\Theta V \Sigma \Sigma^{\dagger}\right)\left[\begin{array}{c}
I_{r} \\
0 \\
0
\end{array}\right]-\mathcal{Z}\left(I_{\ell+p}-\Sigma V\right)\left(I_{\ell+p}-\Sigma \Sigma^{\dagger}\right)\left[\begin{array}{c}
I_{r} \\
0 \\
0
\end{array}\right] \\
& =\mathcal{F}+\Theta \mathcal{G}-\mathcal{Z}\left(I_{\ell+p}-\Sigma V\right) \mathcal{G}
\end{aligned}
$$

where $V \in \mathbb{R}^{n \times \ell+p}$ is an arbitrary matrix. The same development can be made for matrices $\Gamma_{1}$ and $\Gamma_{2}$ and the estimation error dynamics becomes

$$
e(t+1)=(\widetilde{F}-\mathcal{Z} \widetilde{G}) e(t)+\left(\widetilde{F}_{1}-\mathcal{Z} \widetilde{G}_{1}\right) w(t)+\left(\widetilde{F}_{2}-\mathcal{Z} \widetilde{G}_{2}\right) w(t+1)
$$

where

$$
\begin{aligned}
\widetilde{F} & =\mathcal{F}+\Theta V \mathcal{G}, & \widetilde{G} & =\left(I_{\ell+p}-\Sigma V\right) \mathcal{G}, \\
\widetilde{F}_{1} & =\mathcal{F}_{1}+\Theta V \mathcal{G}_{1}, & \widetilde{G}_{1} & =\left(I_{\ell+p}-\Sigma V\right) \mathcal{G}_{1}, \\
\widetilde{F}_{2} & =\mathcal{F}_{2}+\Theta V \mathcal{G}_{2}, & \widetilde{G}_{2} & =\left(I_{\ell+p}-\Sigma V\right) \mathcal{G}_{2},
\end{aligned}
$$

which can be also written as

$$
e(t+1)=(\mathcal{F}-\widetilde{\mathcal{Z}} \mathcal{G}) e(t)+\left(\mathcal{F}_{1}-\widetilde{\mathcal{Z}} \mathcal{G}_{1}\right) w(t)+\left(\mathcal{F}_{2}-\widetilde{\mathcal{Z}} \mathcal{G}_{2}\right) w(t+1)
$$

where

$$
\widetilde{\mathcal{Z}}=-\Theta V+\mathcal{Z}\left(I_{\ell+p}-\Sigma V\right) .
$$

is the new parameter matrix which must be determined, thus the design of the filter is independent of the choice of the generalized inverse.

\subsection{Case where matrix $\Gamma_{2}=0$}

When $\Gamma_{2}=0$ the dynamics of the estimation error (18) becomes

$$
e(t+1)=N e(t)+\Gamma_{1} w(t) .
$$

which is less general then (18) and is independent of $w(t+1)$, then it is free from the temporal correlation of the disturbance $w(t)$. The constraint $\Gamma_{2}=0$ or equivalently $\mathbf{K}_{1} J_{4} D=J_{2} D$ introduces an additional constraint on the parameter matrix $\mathbf{K}_{1}$. When the matrix $D$ is of full row rank, this constraint becomes $\mathbf{K}_{1} J_{4}=J_{2}$, which leads to $\widehat{z}(t)=\zeta(t)$ and the filter(15) becomes

$$
\widehat{z}(t+1)=N \widehat{z}(t)+\Gamma y(t)
$$

a predictor filter, which is exactly the one proposed by [10].

Now the constraint $\mathbf{K}_{1} J_{4} D=J_{2} D$ inserted in (17) leads to the following equation

$$
\left[\begin{array}{lll}
N & \mathbf{K}_{1} & \mathbf{K}_{2}
\end{array}\right] \Sigma_{1}=\Theta_{1}
$$


where $\Sigma_{1}=\left[\begin{array}{cc}L & 0 \\ J_{3} A & J_{4} D \\ C & 0\end{array}\right]$ and $\Theta_{1}=\left[\begin{array}{ll}J_{1} A & J_{2} D\end{array}\right]$.

The necessary and sufficient condition for the existence of the solution to (36) is given by

$$
\operatorname{rank}\left[\begin{array}{c}
\Sigma_{1} \\
\Theta_{1}
\end{array}\right]=\operatorname{rank} \Sigma_{1}
$$

or equivalently

$$
\operatorname{rank}\left[\begin{array}{cc}
L & 0 \\
J_{1} A & J_{2} D \\
J_{3} A & J_{4} D \\
C & 0
\end{array}\right]=\operatorname{rank}\left[\begin{array}{cc}
L & 0 \\
J_{3} A & J_{4} D \\
C & 0
\end{array}\right]
$$

When matrix $D$ is of full row rank, condition (37) becomes rank $\left[\begin{array}{cc}L & 0 \\ J_{1} A & J_{2} \\ J_{3} A & J_{4} \\ C & 0\end{array}\right]=\operatorname{rank}\left[\begin{array}{cc}L & 0 \\ J_{3} A & J_{4} \\ C & 0\end{array}\right]$. Under condition (37), the general solution to (36) is given by

$$
\left[\begin{array}{lll}
N & \mathbf{K}_{1} & \mathbf{K}_{2}
\end{array}\right]=\Theta_{1} \Sigma_{1}^{\dagger}-\mathcal{Z}\left(I_{\ell+p}-\Sigma_{1} \Sigma_{1}^{\dagger}\right)
$$

where $\mathcal{Z}$ is an arbitrary matrix of appropriate dimension.

Now define the following matrices,

$$
\mathcal{F}=\Theta_{1} \Sigma_{1}^{\dagger}\left[\begin{array}{c}
I_{r} \\
0 \\
0
\end{array}\right], \mathcal{G}=\left(I_{\ell+p}-\Sigma_{1} \Sigma_{1}^{\dagger}\right)\left[\begin{array}{c}
I_{r} \\
0 \\
0
\end{array}\right], \mathcal{F}_{1}=J_{1} B-\Theta_{1} \Sigma_{1}^{\dagger}\left[\begin{array}{c}
0 \\
J_{3} B \\
D
\end{array}\right], \mathcal{G}_{1}=\left(I_{\ell+p}-\Sigma_{1} \Sigma_{1}^{\dagger}\right)\left[\begin{array}{c}
0 \\
J_{3} B \\
D
\end{array}\right]
$$

then matrices $N$ and $\Gamma_{1}$ can be written as

$$
N=\mathcal{F}-\mathcal{Z G} \text { and } \Gamma_{1}=\mathcal{F}_{1}-\mathcal{Z} \mathcal{G}_{1}
$$

and equation (18) becomes

$$
e(t+1)=(\mathcal{F}-\mathcal{Z G}) e(t)+\left(\mathcal{F}_{1}-\mathcal{Z} \mathcal{G}_{1}\right) w(t) .
$$

The solution of the $\mathcal{H}_{\infty}$ optimal filtering problem for system (12) is then given by the following theorem.

Theorem 3. Under assumption 1, assume that condition (37) is satisfied, then there exists an unbiased filter for system (12) which solve the $\gamma$-suboptimal $\mathcal{H}_{\infty}$ filtering problem if and only if there exists a matrix $\mathcal{P}=\mathcal{P}^{T}>0$ such that

$$
I_{r}-\mathcal{P}<0
$$

and

$$
\left[\begin{array}{cc}
\mathbb{G}\left(I_{r}-\mathcal{P}\right) \mathbb{G}^{T}-\gamma^{2} \mathbb{G}_{1} \mathbb{G}_{1}^{T} & \left(\mathbb{G} \mathcal{F}^{T}+\mathbb{G}_{1} \mathcal{F}_{1}^{T}\right) \mathcal{P} \\
\mathcal{P}\left(\mathcal{F} \mathbb{G}^{T}+\mathcal{F}_{1} \mathbb{G}_{1}^{T}\right) & -\mathcal{P}
\end{array}\right]<0
$$

with $\left[\begin{array}{ll}\mathbb{G} & \mathbb{G}_{1}\end{array}\right]=\left[\begin{array}{l}\mathcal{G}^{T} \\ \mathcal{G}_{1}^{T}\end{array}\right]^{\perp}$

In this case, all parameter matrices $\mathcal{Z}$ are given by

$$
\mathcal{Z}=\mathbb{B}_{R}^{\dagger} \mathbb{K} \mathbb{C}_{L}^{\dagger}+\mathbb{Z}-\mathbb{B}_{R}^{\dagger} \mathbb{B}_{R} \mathbb{Z} \mathbb{C}_{L} \mathbb{C}_{L}^{\dagger}
$$

where

$$
\begin{aligned}
\mathbb{K} & =-\mathbb{R}_{1}^{-1} \mathbb{B}_{L}^{T} \mathbb{S}_{1} \mathbb{C}_{R}^{T}\left(\mathbb{C}_{R} \mathbb{S}_{1} \mathbb{C}_{R}^{T}\right)^{-1}+\mathbb{R}_{1}^{-1} \mathbb{S}_{2}^{1 / 2} \mathbb{R}_{2}\left(\mathbb{C}_{R} \mathbb{S}_{1} \mathbb{C}_{R}^{T}\right)^{-1 / 2} \\
\mathbb{S}_{1} & =\left(\mathbb{B}_{L} \mathbb{R}_{1}^{-1} \mathbb{B}_{L}^{T}-\Pi\right)^{-1}>0
\end{aligned}
$$




$$
\begin{aligned}
\mathbb{S}_{2} & =\mathbb{R}_{1}-\mathbb{B}_{L}^{T}\left(\mathbb{S}_{1}-\mathbb{S}_{1} \mathbb{C}_{R}^{T}\left(\mathbb{C}_{R} \mathbb{S}_{1} \mathbb{C}_{R}^{T}\right)^{-1} \mathbb{C}_{R} \mathbb{S}_{1}\right) \mathbb{B}_{L} \\
{\left[\begin{array}{ll}
\overline{\mathbb{B}} & \Pi \\
\bullet & \overline{\mathbb{C}}
\end{array}\right] } & =\left[\begin{array}{c|ccc}
0 & I_{r}-\mathcal{P} & 0 & \mathcal{F}^{T} P \\
0 & 0 & -\gamma^{2} I_{q} & \mathcal{F}_{1}^{T} P \\
\mathcal{P} & \mathcal{P} \mathcal{F} & \mathcal{P} \mathcal{F}_{1} & -\mathcal{P} \\
\hline \bullet & \mathcal{G} & \mathcal{G}_{1} & 0
\end{array}\right]
\end{aligned}
$$

and $\mathbb{R}_{1}, \mathbb{R}_{2}$ and $\mathbb{Z}$ are arbitrary matrices of appropriate dimensions satisfying $\mathbb{R}_{1}=\mathbb{R}_{1}^{T}>0$ and $\left\|\mathbb{R}_{2}\right\|<1$. Matrices $\mathbb{B}_{L}, \mathbb{B}_{R}, \mathbb{C}_{L}$ and $\mathbb{C}_{R}$ are any full rank matrices such that $\overline{\mathbb{B}}=\mathbb{B}_{L} \mathbb{B}_{R}$ and $\overline{\mathbb{C}}=\mathbb{C}_{L} \mathbb{C}_{R}$.

Proof. From system (39) and from lemma 5, $\left\|T_{w e}\right\|_{\infty}<\gamma$ if and only if there exists a matrix $\mathcal{P}=\mathcal{P}^{T}>0$ such that

$$
\left[\begin{array}{cc}
(\mathcal{F}-\mathcal{Z} \mathcal{G}) & \left(\mathcal{F}_{1}-\mathcal{Z} \mathcal{G}_{1}\right) \\
I_{r} & 0
\end{array}\right]^{T}\left[\begin{array}{cc}
\mathcal{P} & 0 \\
0 & I_{r}
\end{array}\right]\left[\begin{array}{cc}
(\mathcal{F}-\mathcal{Z} \mathcal{G}) & \left(\mathcal{F}_{1}-\mathcal{Z} \mathcal{G}_{1}\right) \\
I_{r} & 0
\end{array}\right]<\left[\begin{array}{cc}
\mathcal{P} & 0 \\
0 & \gamma^{2} I_{q}
\end{array}\right]
$$

The above inequality can be written as, by using the Schur lemma,

$$
\left[\begin{array}{cccc}
-\mathcal{P} & 0 & (\mathcal{F}-\mathcal{Z} \mathcal{G})^{T} & I_{r} \\
0 & -\gamma^{2} I_{q} & \left(\mathcal{F}_{1}-\mathcal{Z} \mathcal{G}_{1}\right)^{T} & 0 \\
(\mathcal{F}-\mathcal{Z G}) & \left(\mathcal{F}_{1}-\mathcal{Z} \mathcal{G}_{1}\right) & -\mathcal{P}^{-1} & 0 \\
I_{r} & 0 & 0 & -I_{r}
\end{array}\right]<0
$$

By pre- and post-multiplying this inequality by $\left[\begin{array}{cccc}I_{r} & 0 & 0 & 0 \\ 0 & I_{q} & 0 & 0 \\ 0 & 0 & \mathcal{P} & 0 \\ 0 & 0 & 0 & I_{r}\end{array}\right]$, and by applying the Schur lemma we obtain the following inequality

$$
\left[\begin{array}{ccc}
I_{r}-\mathcal{P} & 0 & (\mathcal{F}-\mathcal{Z G})^{T} \mathcal{P} \\
0 & -\gamma^{2} I_{q} & \left(\mathcal{F}_{1}-\mathcal{Z} \mathcal{G}_{1}\right)^{T} \mathcal{P} \\
\mathcal{P}(\mathcal{F}-\mathcal{Z G}) & \mathcal{P}\left(\mathcal{F}_{1}-\mathcal{Z} \mathcal{G}_{1}\right) & -\mathcal{P}
\end{array}\right]<0
$$

which can be also written as

$$
\Pi-\left[\begin{array}{c}
0 \\
0 \\
\mathcal{P}
\end{array}\right] \mathcal{Z}\left[\begin{array}{lll}
\mathcal{G} & \mathcal{G}_{1} & 0
\end{array}\right]-\left[\begin{array}{lll}
\mathcal{G} & \mathcal{G}_{1} & 0
\end{array}\right]^{T} \mathcal{Z}^{T}\left[\begin{array}{lll}
0 & 0 & \mathcal{P}
\end{array}\right]<0
$$

where

$$
\Pi=\left[\begin{array}{ccc}
I_{r}-\mathcal{P} & 0 & \mathcal{F}^{T} \mathcal{P} \\
0 & -\gamma^{2} I_{q} & \mathcal{F}_{1}^{T} \mathcal{P} \\
\mathcal{P} \mathcal{F} & \mathcal{P} \mathcal{F}_{1} & -\mathcal{P}
\end{array}\right]
$$

Using lemma 6 , this matrix inequality is solvable for $\mathcal{Z}$ if and only if

$$
\left[\begin{array}{l}
0 \\
0 \\
\mathcal{P}
\end{array}\right]^{\perp} \Pi\left[\begin{array}{l}
0 \\
0 \\
\mathcal{P}
\end{array}\right]^{T \perp}<0
$$

and

$$
\left[\begin{array}{lll}
\mathcal{G} & \mathcal{G}_{1} & 0
\end{array}\right]^{T \perp} \Pi\left[\begin{array}{lll}
\mathcal{G} & \mathcal{G}_{1} & 0
\end{array}\right]^{T \perp T}<0 .
$$

Inequality (40) is satisfied since

$$
\left[\begin{array}{l}
0 \\
0 \\
\mathcal{P}
\end{array}\right]^{\perp}=\left[\begin{array}{ccc}
I_{r} & 0 & 0 \\
0 & I_{q} & 0
\end{array}\right]
$$


and inequality (47) is exactly (41) since

$$
\left[\begin{array}{c}
\mathcal{G}^{T} \\
\mathcal{G}_{1}^{T} \\
0
\end{array}\right]^{\perp}=\left[\begin{array}{ccc}
\mathbb{G} & \mathbb{G}_{1} & 0 \\
0 & 0 & I_{r}
\end{array}\right]
$$

with $\left[\begin{array}{ll}\mathbb{G} & \mathbb{G}_{1}\end{array}\right]=\left[\begin{array}{l}\mathcal{G}^{T} \\ \mathcal{G}_{1}^{T}\end{array}\right]^{\perp}$

Conditions (40)-(41) are linear matrix inequalities (LMIs) on the variable $\mathcal{P}$, that is, the unbiased $\gamma$ suboptimal $\mathcal{H}_{\infty}$ reduced order filtering problem is an LMI problem. The following remark gives a solution of this problem without the parametrization of all the solutions as in theorem 3.

Remark 4. The solution of the above $\mathcal{H}_{\infty}$ problem can be obtained directly from the solution of (44) or equivalently by solving

$$
\left[\begin{array}{ccc}
I_{r}-\mathcal{P} & 0 & \mathcal{F}^{T} \mathcal{P}-\mathcal{G}^{T} \mathcal{Y}^{T} \\
0 & -\gamma^{2} I_{q} & \mathcal{F}_{1}^{T} \mathcal{P}-\mathcal{G}_{1}^{T} \mathcal{Y}^{T} \\
\mathcal{P} \mathcal{F}-\mathcal{Y} \mathcal{G} & \mathcal{P} \mathcal{F}_{1}-\mathcal{Y} \mathcal{G}_{1} & -\mathcal{P}
\end{array}\right]<0
$$

The parameter matrix $\mathcal{Z}$ is then given by $\mathcal{Z}=\mathcal{P}^{-1} \mathcal{Y}$.

\subsection{Connection with the standard case}

Consider system (12) with $E=I_{n}$. In this case one can see that one choice of the matrix $J$ is $J=$ $\left[\begin{array}{ll}J_{1} & J_{2} \\ J_{3} & J_{4}\end{array}\right]=\left[\begin{array}{cc}L & 0 \\ -C & I_{p}\end{array}\right]$, which leads to $J\left[\begin{array}{l}I_{n} \\ C\end{array}\right]=\left[\begin{array}{l}L \\ 0\end{array}\right]$. Then the filter (15) becomes

$$
\begin{aligned}
& \zeta(t+1)=N \zeta(t)+\bar{\Gamma} u(t)+\Gamma y(t) \\
& \widehat{z}(t)=\zeta(t)-\mathbf{K}_{1} y(t)
\end{aligned}
$$

where $\widehat{z}(t) \in \mathbb{R}^{r}$ is the estimate of $z(t)$.

From the above results, condition (19) can be written as rank $\left[\begin{array}{c}L \\ L A \\ C A \\ C\end{array}\right]=\operatorname{rank}\left[\begin{array}{c}L \\ C A \\ C\end{array}\right]$ which corresponds to that given by [17] which is more general than the condition given by [10]. When $\Gamma_{2}=0$, from (36) we obtain $\mathbf{K}_{1} D=0$ and condition (37) becomes $\operatorname{rank}\left[\begin{array}{cc}L & 0 \\ L A & 0 \\ C A & D \\ C & 0\end{array}\right]=\operatorname{rank}\left[\begin{array}{cc}L & 0 \\ C A & D \\ C & 0\end{array}\right]$ which is also satisfied when the condition given in [10] is satisfied but not the converse. When matrix $D$ is of full row rank we obtain $\mathbf{K}_{1}=0$. In this case we obtain the condition of [10] and theorem 4 corresponds to theorem 3 of [10]. These remarks reveal that the condition given in this paper are weaker than those adopted in [10].

\section{Numerical example}

To illustrate our results, let us consider the electromechanical actuator described in [18] and constituting of a direct current motor with an elastic coupling and the load shaft as shown in figure 1. This plant can be described by the following state-space model

$$
\begin{aligned}
& \dot{x}_{m}=A_{c} x_{m}+R_{c} u+M_{c} d+B_{c} w \\
& y=C_{c} x_{m}+D_{c} w
\end{aligned}
$$


where the index ' $c$ ' stands for continuous time and

$$
\begin{aligned}
A_{c} & =\left[\begin{array}{ccc}
\frac{-F_{m}}{J_{m}} & \frac{-k_{t}}{N_{1} J_{m}} & 0 \\
\frac{1}{N_{1}} & 0 & -1 \\
0 & \frac{k_{t}}{J_{c}} & \frac{-F_{c}}{J_{c}}
\end{array}\right], R_{c}=\left[\begin{array}{c}
\frac{k_{a}}{J_{m}} \\
0 \\
0
\end{array}\right], M_{c}=\left[\begin{array}{c}
0 \\
0 \\
-1 \\
J_{c}
\end{array}\right], B_{c}=\left[\begin{array}{cc}
\frac{k_{a}}{J_{m}} & 0 \\
0 & 0 \\
0 & 0
\end{array}\right], C_{c}=\left[\begin{array}{ccc}
1 & 0 & 0 \\
0 & 0 & 1
\end{array}\right], \\
D_{c} & =\left[\begin{array}{cc}
0 & 0 \\
0 & 0.5
\end{array}\right], x_{m}=\left[\begin{array}{c}
\omega_{m} \\
\Delta_{\Theta} \\
\omega_{c}
\end{array}\right] .
\end{aligned}
$$

The state variables are the motor shaft velocity $\omega_{m}$, the elastic torque $\Delta_{\Theta}$ and the motor shaft velocity $\omega_{c}$. The control input $u$ is the stator current $i_{e}$. The unknown disturbance $d$ is due to Coulomb frictions and load disturbance. The vector $w$ represents finite energy disturbances which affect both the control input and the second measurement. In the state-space description, $J_{m}$ and $J_{c}$ represent the motor and the load shaft inertia, $F_{m}$ and $F_{c}$ the motor and the load viscous friction coefficients, $k_{a}$ the motor torque constant, $k_{t}$ the coupling rigidity coefficient and $N_{1}$ the gear ratio. The numerical values of these parameters are $N_{1}=20, k_{a}=0.156\left[\mathrm{~m}^{2} \mathrm{~kg} \mathrm{sec}{ }^{-2} \mathrm{~A}^{-2}\right], k_{t}=37.7\left[\mathrm{~m}^{2} \mathrm{~kg} \mathrm{sec}^{-2}\right], F_{m}=0.0032\left[\mathrm{~m}^{2} \mathrm{~kg}\right.$ $\left.\mathrm{sec}^{-1}\right], F_{c}=0\left[\mathrm{~m}^{2} \mathrm{kgsec}^{-1}\right], J_{m}=0.00024\left[\mathrm{~m}^{2} \mathrm{~kg}\right]$ and $J_{c}=0.0825\left[\mathrm{~m}^{2} \mathrm{~kg}\right]$.

The objective is to estimate, simultaneously, the unmeasured elastic torque $\Delta_{\Theta}$ and the unknown input $d$ via a reduced order filter.

The continuous-time model is discretized using the 'forward rectangular' method (see function 'bilin' with option 'FwdRec' in Matlab ${ }^{\circledR}$ ) with a time period $T=0.001$ seconde. We obtain the following discrete-time model

$$
\begin{aligned}
& x_{m}(t+1)=A_{d} x_{m}(t)+R_{d} u(t)+M_{d} d(t)+B_{d} w(t) \\
& y(t)=C_{d} x_{m}(t)+D_{d} w(t)
\end{aligned}
$$

with

$$
\begin{aligned}
A_{d} & =\left[\begin{array}{ccc}
0.9867 & -7.8542 & 0 \\
0.0001 & 1 & -0.001 \\
0 & 0.4570 & 1
\end{array}\right], R_{d}=\left[\begin{array}{c}
0.65 \\
0 \\
0
\end{array}\right], M_{d}=\left[\begin{array}{c}
0 \\
0 \\
0.0121
\end{array}\right], B_{d}=\left[\begin{array}{cc}
0.001 & 0 \\
0 & 0 \\
0 & 0
\end{array}\right], C_{d}=\left[\begin{array}{lll}
1 & 0 & 0 \\
0 & 0 & 1
\end{array}\right], \\
D_{d} & =\left[\begin{array}{cc}
0 & 0 \\
0 & 0.5
\end{array}\right] .
\end{aligned}
$$

To estimate the state $\Delta_{\Theta}$ and the unknown input $d$ for system (51), this system can be written in the following discrete-time descriptor system $[5,19]$

$$
\begin{aligned}
E x(t+1) & =A x(t)+R u(t)+B w(t) \\
y(t) & =C x(t)+D w(t) \\
z(t) & =L x(t)
\end{aligned}
$$

where

$$
E=\left[\begin{array}{ll}
I_{3} & 0
\end{array}\right], A=\left[\begin{array}{ll}
A_{d} & M_{d}
\end{array}\right], R=R_{d}, B=B_{d}, C=\left[\begin{array}{ll}
C_{d} & 0
\end{array}\right], D=D_{d}, L=\left[\begin{array}{llll}
0 & 1 & 0 & 0
\end{array}\right], x=\left[\begin{array}{c}
x_{m} \\
d
\end{array}\right] .
$$

For this example, assumption 1 and conditions (19) and (37) are satisfied where matrix $J$ defined in equation (14) can be chosen as $J=\left[\begin{array}{ll}J_{1} & J_{2} \\ J_{3} & J_{4}\end{array}\right]=\left[\begin{array}{ccc|cc}0 & 1 & 0 & 0 & 0 \\ \hline-1 & 0 & 0 & 1 & 0 \\ 0 & 0 & -1 & 0 & 1\end{array}\right]$ and $\ell=m+p+r-\operatorname{rank}\left[\begin{array}{l}E \\ C\end{array}\right]=3$. 
From the results of section 2 we obtain the following matrices.

$$
\begin{aligned}
& \mathcal{F}=0.0310, G=\left[\begin{array}{c}
0.9690 \\
-0.1234 \\
0 \\
-0.1217 \\
0
\end{array}\right], \mathcal{F}_{1}=\left[\begin{array}{ll}
0.0802 & 0.0005
\end{array}\right], \mathcal{G}_{1}=\left[\begin{array}{cc}
0.0802 & 0 \\
-0.0102 & 0 \\
0 & 0 \\
-0.0101 & 0 \\
0 & 0
\end{array}\right], \mathcal{F}_{2}=\left[\begin{array}{ll}
0 & 0
\end{array}\right], \mathcal{G}_{2}=\left[\begin{array}{ll}
0 & 0 \\
0 & 0 \\
0 & 0 \\
0 & 0 \\
0 & 0
\end{array}\right], \\
& \overline{\mathcal{G}}=\mathcal{G}, \overline{\mathcal{G}}_{1}=\mathcal{G}_{1}, \mathbb{K}_{1}=0, \mathbb{K}_{2}=\left[\begin{array}{ll}
0 & 1
\end{array}\right], \gamma=0.1, P_{1}=14.0743, Q_{1}=13.6830, Q_{3}=\left[\begin{array}{cc}
12.5193 & 0 \\
0 & 11.5199
\end{array}\right] \text {, }
\end{aligned}
$$

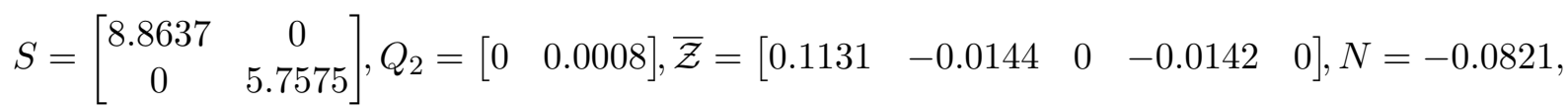

$$
\begin{aligned}
& \Gamma=\left[\begin{array}{ll}
0.1473 & -0.001
\end{array}\right], \mathbf{K}_{1}=\left[\begin{array}{ll}
0.1378 & 0
\end{array}\right], \mathbf{K}_{2}=\left[\begin{array}{ll}
0.1360 & -0.001
\end{array}\right], \bar{\Gamma}=0.0896, \Gamma_{1}=\left[\begin{array}{ll}
0.0708 & 0.0005
\end{array}\right] \text { and } \\
& \Gamma_{2}=\left[\begin{array}{ll}
0 & 0
\end{array}\right] \text {. }
\end{aligned}
$$

The optimal $\mathcal{H}_{\infty}$ norm was found to be $\gamma=0.1$. Then the following $\mathcal{H}_{\infty}$ filter was obtained

$$
\begin{aligned}
& \zeta(t+1)=-0.0821 \zeta(t)+0.0896 u(t)+\left[\begin{array}{ll}
0.1473 & -0.001
\end{array}\right] y(t) \\
& \widehat{z}(t)=\zeta(t)-\left[\begin{array}{ll}
0.1378 & 0
\end{array}\right] y(t)
\end{aligned}
$$

The state responses are depicted in figure 3 and the disturbances and the control input are shown in figure 2. The functional $z(t)$ and its estimation $\widehat{z}(t)$ are plotted in figure 4 . The estimation error for the estimate $\hat{z}(t)$ is presented in figure 5 which shows the performances of the proposed approach.

\section{Conclusion}

In this paper, a new method for the unbiased reduced order $\mathcal{H}_{\infty}$ filter design for descriptor systems has been developed. The obtained results generalize those presented in $[4,9,10]$. The necessary and sufficient conditions for the existence of the filter are given from LMIs formulation.

\section{References}

[1] D. Luenberger, "Dynamic equations in descriptor form," IEEE Trans. Aut. Contr., vol. 32, pp. 312$321,1977$.

[2] J. Mills and A. Goldenberg, "Force and position control of manipulators during constrained motion tasks," IEEE Trans. Rob. Autom., vol. 68, pp. 30-46, 1989.

[3] L. Dai, Singular Control Systems, vol. 118 of Lecture Notes in Control and Information Sciences. New York: Springer-Verlag, 1989.

[4] R. Nikoukhah, A. Willsky, and B. Levy, "Kalman filtering and Riccati equations for descriptor systems," IEEE Trans. Aut. Contr., vol. 37, pp. 1325-1342, 1992.

[5] M. Darouach, M. Zasadzinski, and D. Mehdi, "State estimation of stochastic singular linear systems," Int. J. Syst. Sci., vol. 24, pp. 345-354, 1993.

[6] M. Darouach, M. Zasadzinski, and A. Bassong Onana, "Connection between the 3-block generalized Riccati equation and the standard Riccati equation," IEEE Trans. Aut. Contr., vol. 39, pp. 17551758, 1994.

[7] R. Nikoukhah, S. Campbell, and F. Delebecque, "Kalman filtering for general discrete-time systems," IEEE Trans. Aut. Contr., vol. 44, pp. 1829-1839, 1999. 
[8] K. Nagpal and P. Khargonekar, "Filtering and smoothing in an $\mathcal{H}_{\infty}$ setting," IEEE Trans. Aut. Contr., vol. 36, pp. 152-166, 1991.

[9] M. Darouach, "On the optimal unbiased functional filtering," IEEE Trans. Aut. Contr., vol. 45, pp. 1374-1379, 2000.

[10] J. Watson and K. Grigoriadis, "Optimal unbiased filtering via linear matrix inequalities," Syst. E Contr. Letters, vol. 35, pp. 111-118, 1998.

[11] S. Xu and J.Lam, "Reduced-order $\mathcal{H}_{\infty}$ filtering for singular systems," Syst. $\&$ Contr. Letters, vol. 56, pp. 48-57, 2007.

[12] G. Zhang, Y. Xia, and P. Shi, "New bounded real lemma for discrete-time singular systems," Automatica, vol. 44, pp. 886-890, 2008.

[13] C. Rao and S. Mitra, Generalized Inverse of Matrices and its Applications. New York: Wiley, 1971.

[14] R. Skelton, T. Iwasaki, and K. Grigoriadis, A Unified Algebraic Approach to Linear Control Design. London: Taylor \& Francis, 1998.

[15] T. Iwasaki and R. Skelton, "All controllers for the general $\mathcal{H}_{\infty}$ control problems : LMI existence conditions and state space formulas," Automatica, vol. 30, pp. 1307-1317, 1994.

[16] S. Xu and C. Yang, "Stabilization of discrete-time singular systems : a matrix inequalities approach," Automatica, vol. 35, pp. 1613-1617, 1999.

[17] M. Darouach, "Existence and design of functional observers for linear systems," IEEE Trans. Aut. Contr., vol. 45, pp. 940-943, 2000.

[18] O. Malassé, M. Zasadzinski, C. Iung, M. Hayar, and M. Darouach, "Comparison of robust loop shaping designs : an application to an electromechanical actuator," in Proc. IEEE Conf. Contr. E Applications, (Glasgow, UK), 1994.

[19] M. Darouach, M. Zasadzinski, A. Bassong Onana, and S. Nowakowski, "Kalman filtering with unknown inputs via optimal state estimation of singular systems," Int. J. Syst. Sci., vol. 26, pp. 20152028, 1995.

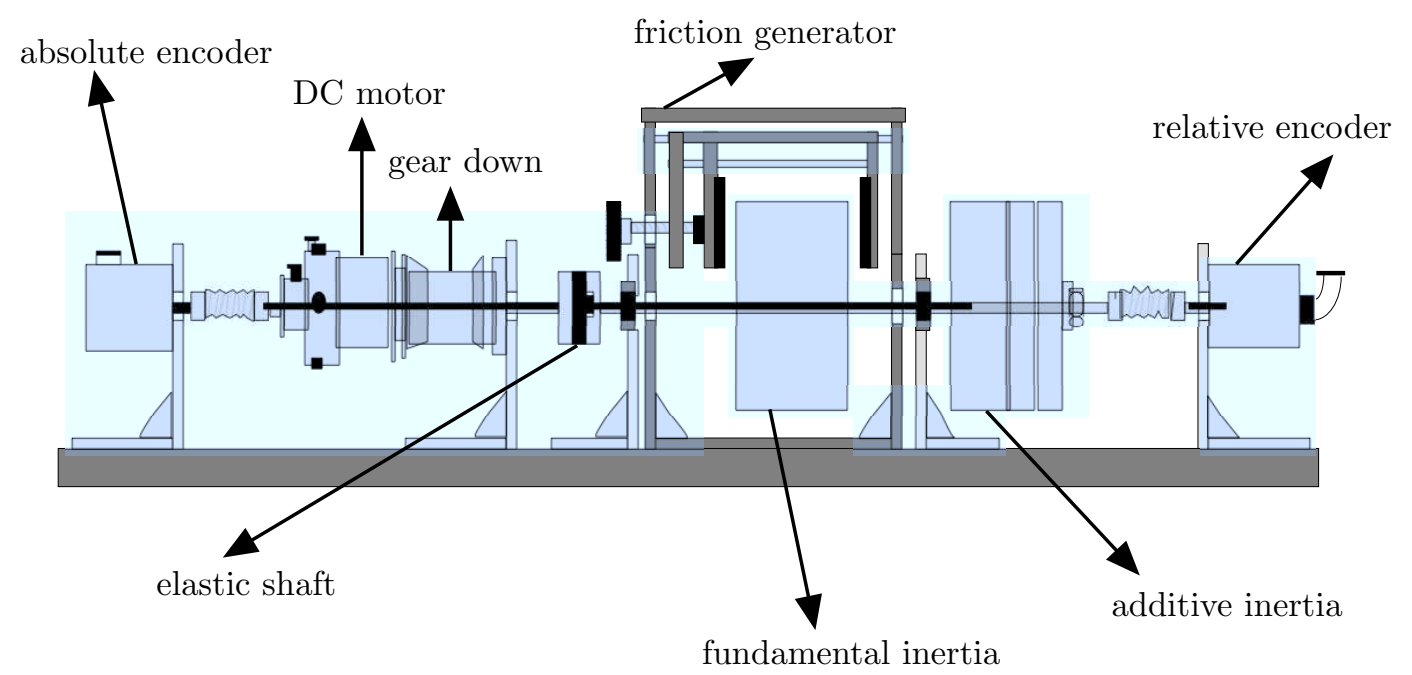

Figure 1: Electromechanical actuator. 


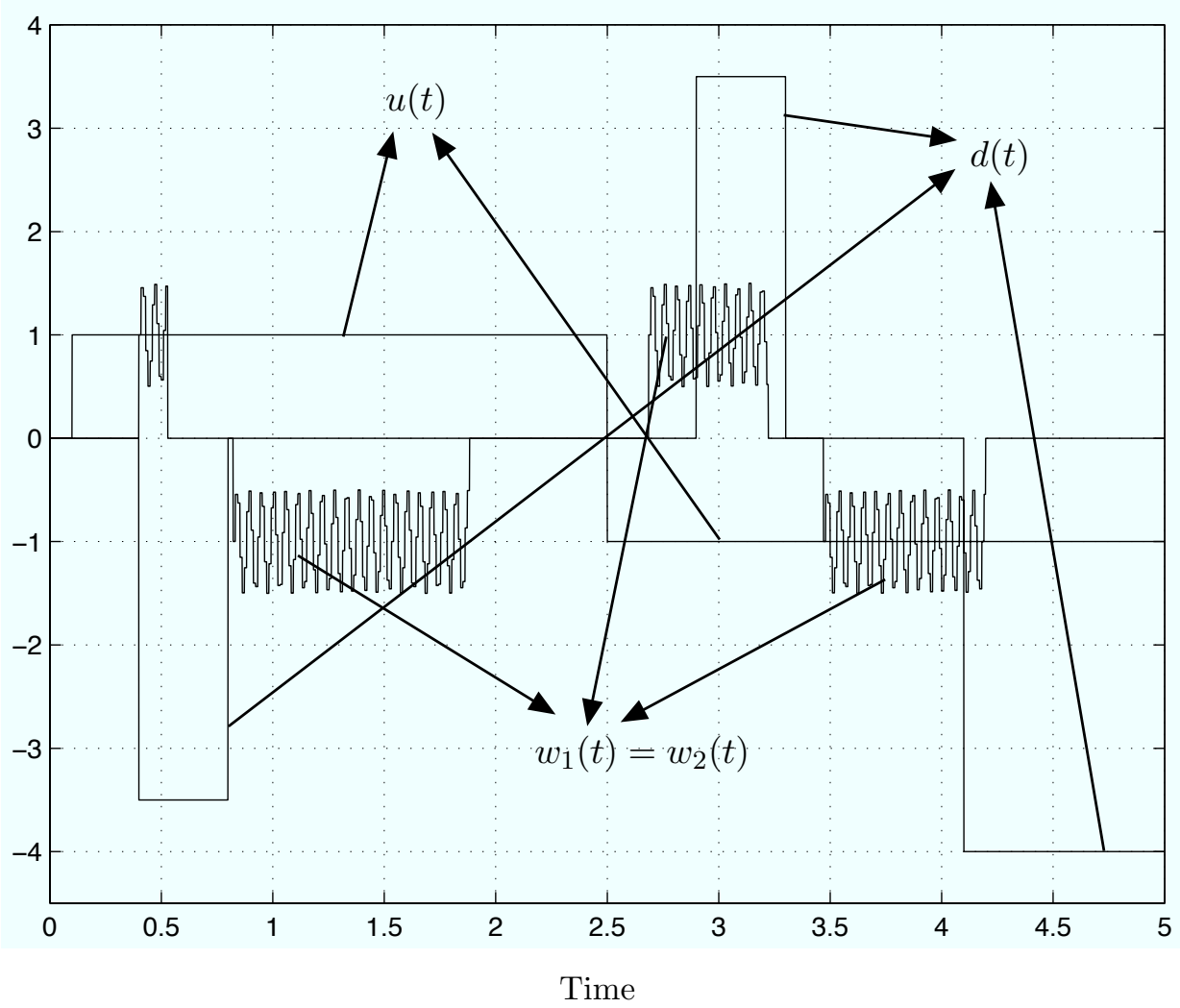

Figure 2: The control input $u(t)$, the unknown disturbance $d(t)$, and the finite energy disturbances $w(t)$.

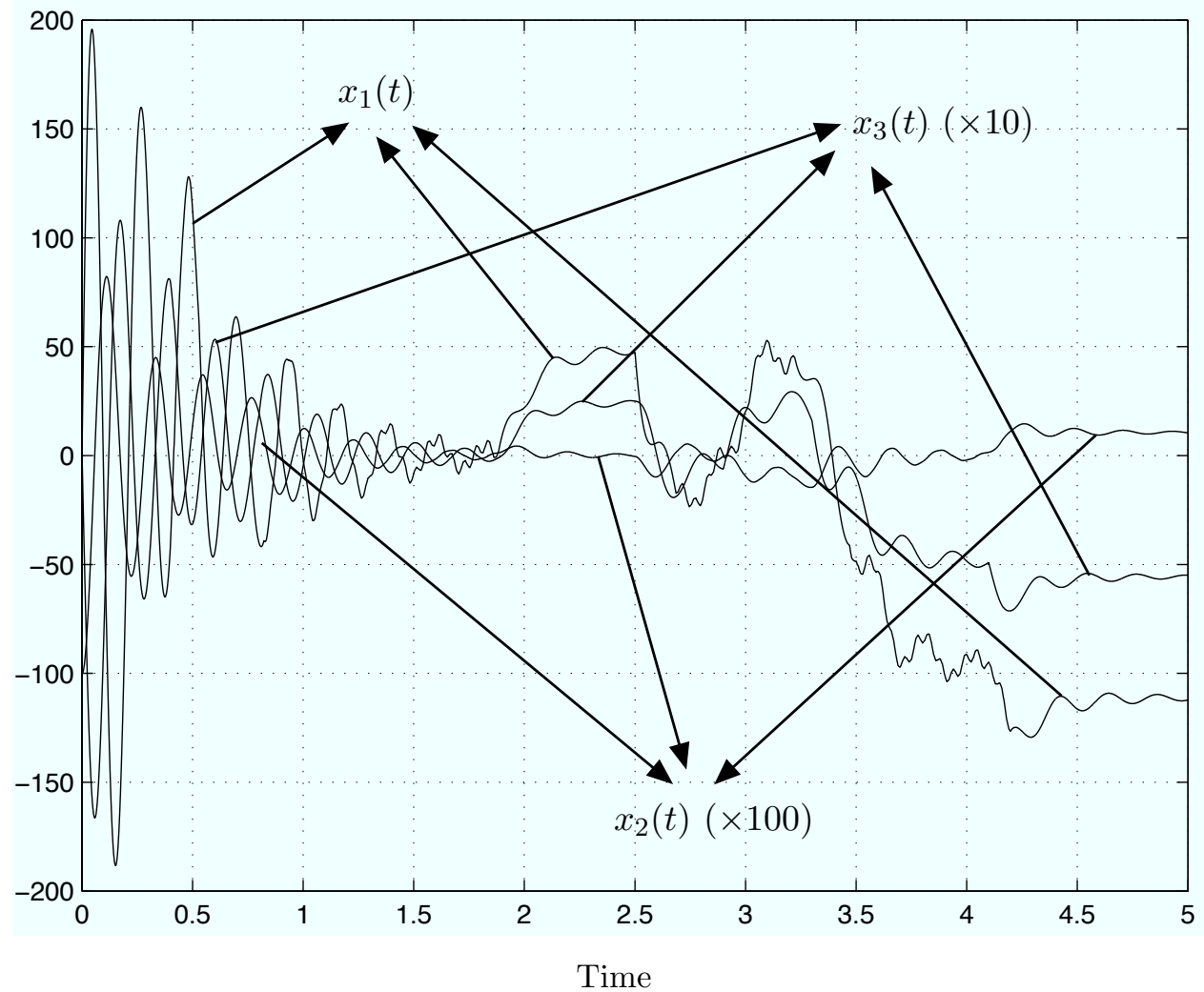

Figure 3: The actual states $x(t)$. 


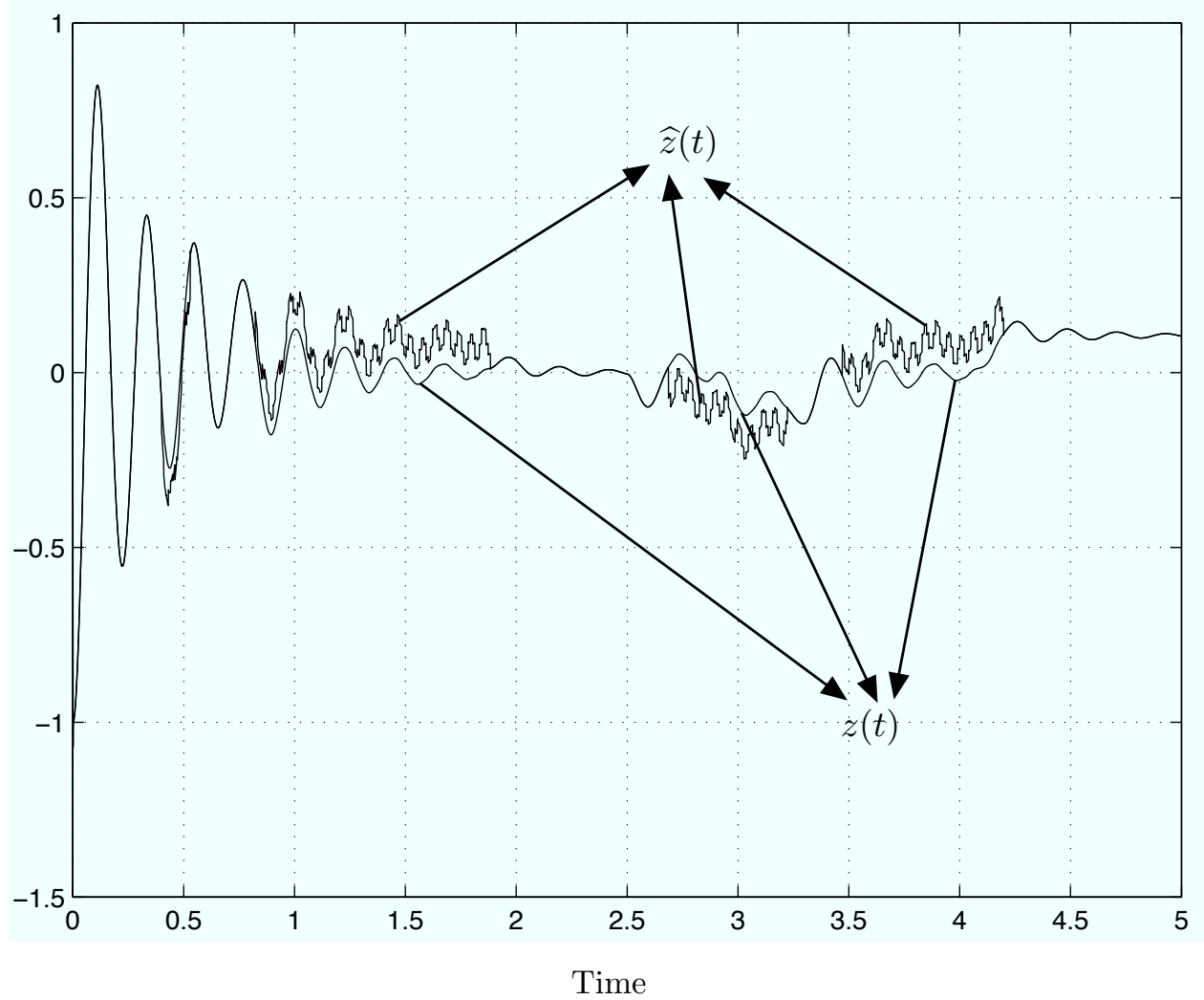

Figure 4: The functional $z(t)=x_{2}(t)$ and the estimated functional $\widehat{z}(t)$.

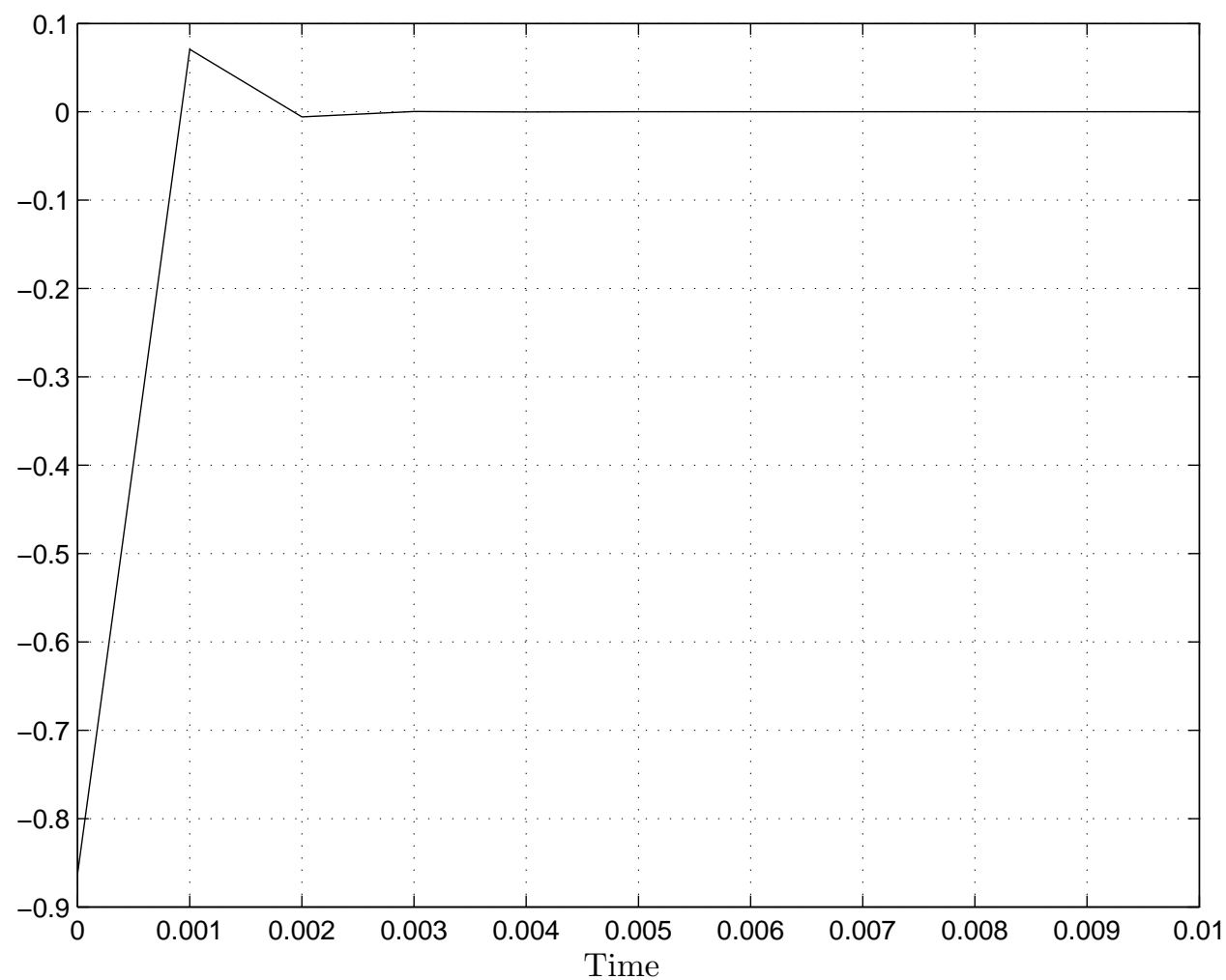

Figure 5: Estimation error $e(t)$. 